\title{
Predisposing, Enabling, and Need-Related Factors Associated with Human Papillomavirus Vaccination Intentions and Uptake Among Black and Hispanic Sexual and Gender Diverse Adults in the USA
}

\author{
Christopher W. Wheldon ${ }^{1}$ (D) - Lisa A. Eaton ${ }^{2} \cdot$ Ryan J. Watson ${ }^{2}$
}

Received: 22 October 2021 / Revised: 14 December 2021 / Accepted: 15 December 2021 / Published online: 3 January 2022

(c) W. Montague Cobb-NMA Health Institute 2022

\begin{abstract}
The purpose of the current study was to identify predisposing, enabling, and need-based factors associated with human papillomavirus (HPV) or HPV vaccination intentions among ethnoracial minority men who have sex with men (MSM) and gender expansive natal males. This was a secondary analysis of survey data from 299 Black and Hispanic MSM and gender expansive young (aged 18-30 years) adults living in the USA. Variable selection was informed by Anderson's model of healthcare utilization. Outcomes were self-reported HPV vaccination and vaccine intentions. Less than half of the sample (45.5\%) reported initiating (i.e., receiving at least 1 dose) HPV vaccination. Hierarchical multiple regression was used to identify relevant theoretical predictors. Among those who were unvaccinated, $14.2 \%$ were unlikely, $56.3 \%$ were undecided, and $29.4 \%$ likely to initiate HPV vaccination within the next 12 months. More favorable vaccine attitudes (adjusted prevalence ratio $[a P R]=1.55 ; 95 \% C I: 1.12-2.15)$, past year routine check-up $(a P R=1.50 ; 95 \% C I: 1.14-1.97)$, and ever being diagnosed with anogenital warts $(a P R=1.55 ; 95 \% C I: 1.12-2.15)$ were independently associated with the probability of HPV vaccination. Several enabling factors (e.g., testing for sexually transmitted infections) were not associated with HPV vaccination, suggesting routine missed opportunities. There were no associations between predictor variables and HPV vaccine intentions. Targeted efforts are needed to decrease anal cancer disparities experienced by ethnoracial minority MSM. Leveraging enabling factors already present this population (e.g., STI testing and pre-exposure prophylaxis use) are potential targets for interventions to increase the reach of HPV vaccination.
\end{abstract}

Keywords Vaccines $\cdot$ SGM $\cdot$ HPV intervention $\cdot$ MSM

\section{Introduction}

Anal cancer, while rare in the general population, disproportionately affects men who have sex with men (MSM), in addition to populations with high prevalence of anal human papilloma virus (HPV) infections (e.g., transgender women who have sex with men) [1]. African American men, particularly men $<50$ years old, have an increased risk of anal cancer [2-4]. Black race was also found to be a poor

Christopher W. Wheldon

Chris.wheldon@temple.edu

1 Department of Social and Behavioral Sciences, College of Public Health, Temple University, 1301 Cecil B. Moore Ave., Ritter Hall Annex 9th Floor, Room 955, Philadelphia, PA, USA

2 Department of Human Development and Family Sciences, University of Connecticut, Storrs, CT, USA prognostic factor in anal cancer specific survival, independent of socioeconomic status [5].

Like cervical cancer, persistent HPV infection is the necessary cause of anal cancers [1]. Anal sex behaviors (e.g., receptive anal intercourse) are a significant risk factor for HPV infection and subsequent anal cancer. However, unlike for cervical cancer, there are no national guidelines regarding anal cancer screening [6]. Thus, current public health efforts emphasize primary prevention through prophylactic HPV vaccination [7].

Vaccination against HPV types 16 and 18, either through quadrivalent or nonavalent HPV vaccination, is safe and effective at preventing anal cancer [8]. While HPV vaccination is prioritized for adolescents aged 11 and 12, catch-up vaccination is recommended up through age 26 [7]. HPV vaccination coverage among adolescent males is currently insufficient $(\sim 52 \%)$ to reduce anal cancer disparities [9]; thus, targeted interventions to increase reach and uptake 
among those most at risk are required. In one nationally representative study, HPV vaccine initiation was only $32.8 \%$ among MSM aged $18-26$ and $13.2 \%$ among those was 27 years and older [10]. Coverage is similar among transgender women, but lower among Black and Hispanic MSM $[10,11]$.

Research is needed to identify factors associated with HPV vaccine uptake among ethnoracial MSM for three primary reasons. First, African American men have higher risk of anal cancer development and are more likely to experience poorer treatment outcomes. Second, HPV vaccination uptake among Black and Hispanic MSM is lower than their non-Hispanic counterparts. Third, intersectionality suggests that Black and Hispanic MSM experience interacting systems of oppression that have direct impact on relevant predictors of HPV vaccination, such as their trust in the recommendations of medical authorities and their comfort in engaging with providers regarding stigmatized behaviors (i.e., anal intercourse) [12].

Many of the existing studies on HPV vaccine uptake in MSM do not report on an explicit theoretical approach or rely heavily on intrapersonal theories of health behavior (e.g., the theory of planned behavior) [13]. In the current study, Andersen's model of healthcare utilization was used to identity predisposing (i.e., sociocultural factors that influence HPV vaccine decisions), enabling (i.e., factors that may facilitate utilization of HPV vaccination), and need based factors (i.e., professional judgment about an individual's need for HPV vaccination) associated with HPV vaccination (initiation or intentions) among an at risk sample of Black and Hispanic MSM and gender-expansive young adults [14]. This theoretical framework is frequently used to identify factors that facilitate or impede the utilization of health services. This approach provided the conceptual basis to assess equity in the uptake of HPV vaccination among underserved populations. The following research question was explored: Which predisposing, enabling, and need based factors are associated with HPV vaccination or HPV vaccination intentions among ethnoracial minority MSM and gender expansive natal males?

\section{Methods}

\section{Participants and Procedures}

This was a secondary analysis of data from the second wave of the Longitudinal PrEP and Substance Use National Survey, a panel study to explore sexual health behaviors among Black and Hispanic MSM and gender expansive natal males. The study consisted of 300 participants with two waves of data collection. HPV vaccination was assessed at wave 2 . Wave 2 data was collected between February and March of
2021. The initial inclusion criteria included (1) age 18-29, (2) identify as Black, Hispanic, Latino, and/or Latinx, (3) reported male sex at birth, (4) resided in the USA, and (5) had anal sex with another man in the past year. All study protocols were approved by the University of Connecticut's Institutional Review Board.

Baseline data were collected in partnership with the Human Rights Campaign (HRC), a US-based non-profit LGBTQ advocacy group and political lobbying organization. HRC and researchers contacted national networks, utilized several large mailing lists, and posted on social media (Twitter, Facebook, and Instagram) accounts with the assistance from HRC's wide-reaching network of community partners. Additionally, HRC leveraged connections with local community-based organizations, health departments, and other health centers.

At follow up in wave 2, participants were recontacted and invited to complete an anonymous web-based questionnaire. Most survey instruments were repeated from wave 1, but COVID and HPV questions were newly included. Participants were provided a $\$ 25$ gift card to Amazon, or a Venmo (cash) payment as an incentive.

\section{Measures}

Outcome variables Self-reported HPV vaccination and HPV vaccine intentions (for those unvaccinated) were the main outcome variables. Participants were asked: "Do you plan to get the first HPV vaccine shot in the next 12 months?" Participants that responded, I have already received the $H P V$ vaccine, where coded as vaccinated. Those who were not previously vaccinated responded either Yes, I will [definitely, probably] get the first shot in the next 12 months, I do not know/I am unsure, or No, I will [definitely, probably] NOT get the first shot in the next 12 months. The next 12-month HPV vaccine intentions were coded as likely (definitely or probably will), undecided, and unlikely (definitely or probably will not).

\section{Predisposing factors}

Sociodemographics Predisposing factors included age cohorts based on current HPV vaccine recommendations (18-26 for routine catchup or 27-30 for shared clinical decision making), ethnoracial identity (non-Hispanic Black or Hispanic), educational attainment, and marital status (married or in a domestic partnership vs single, divorced, or widowed). Sexual orientation and gender identity (SOGI) were assessed as sexual identity (gay/same gender loving, bisexual/pansexual, or another identity including heterosexual or straight, queer, questioning, or other) and current gender identity (cis-gender man or gender expansive, which included agender, genderfluid, genderqueer, or nonbinary). 
Vaccination Beliefs Participants were asked, "How likely are you to get vaccinated for coronavirus once a vaccination is available to the public?" for which they responded as either very unlikely, somewhat unlikely, unsure, somewhat likely, or very likely. They were also asked to rate their level of agreement or disagreement on a 4-point Likert scale to the following statements about childhood vaccines: (1) have many known harmful side effects, (2) provides important benefit to society, (3) may lead to illness and death, and (4) are useful and effective [15]. Responses were averaged into a single scale score with higher values indicating more positive attitudes toward vaccines $(\alpha=0.80)$.

\section{Enabling factors}

Health Care Status Enabling factors included health insurance coverage (any kind of coverage vs no coverage), health care utilization (i.e., routine check-up defined as a general physical exam, not an exam for a specific injury, illness, or condition), and being tested for a sexually transmitted infection within the previous year.

Pre-exposure prophylaxis (PrEP) Use Status PrEP use was assessed with two items. The first item asked of participants who reported that they were aware of PrEP: "Have you ever taken PrEP before, even if just one pill?" Response options were No and Yes. Participants who had never heard of PrEP were excluded $(n=5)$. A separate item asked participants who reported they had ever taken PrEP, "Do you currently take PrEP?" with response options of No and Yes. Based on these responses, PrEP use was classified as Never, not current, and current.

\section{Need-Based factors}

Need-based factors (i.e., professional judgment about need for HPV vaccination) included those related to HPV exposure. Participants were asked to indicate if they had ever been diagnosed with anogenital warts and engaged in anal sex with a man within the past month. Response options were No and Yes. If they had ever engaged in anal sex with a man, they were asked "How old were you the first time you had anal sex with a man?" which was entered as a continuous value.

\section{Analysis}

All analyses were conducted in SAS 9.4 (SAS Institute, Cary, NC). Bivariate differences were examined using chisquare test of independence, Fisher's exact test, or analysis of variance, where appropriate. Variables with bivariate associations $(p<0.10)$ with HPV vaccination or HPV vaccine intentions were included in multivariable regression models. Models were run separately for predisposing (model 1 ), enabling (model 2), and need related factors (model 3 ). Variables with statistically significant $(p<0.05)$ independent associations with HPV vaccination in models $1-3$ were included in a final model (model 4). Adjusted prevalence ratios with $95 \%$ confidence intervals were reported as the measure of association. Three hundred participants completed the second wave of the survey; however, one participant was excluded because of a missing response on the HPV vaccination question. Thus, the analytic sample for this study was 299. Listwise deletion was used for the missing data on predictor variables ( $<2 \%$ for any given variable).

\section{Results}

Sample characteristics are reported in Table 1. The average age was $26.5(\mathrm{SD}=2.7)$. Most identified as Hispanic or Latino $(66.2 \%)$, cisgender men $(93.3 \%)$, gay or same gender loving $(80.0 \%)$, and unmarried $(93.0 \%)$. General vaccine attitudes were positive $(\mathrm{M}=3.59 ; \mathrm{SD}=0.49)$ as were COVID-19 vaccine intentions ( $85.6 \%$ likely). The majority had health insurance $(84.6 \%)$, had a routine check within the previous year (77.6\%), and were recently tested for STIs (77.6\%). Current PrEP use was reported by less than half $(42.3 \%)$. The average age for first anal sex with a male partner was $17.6(\mathrm{SD}=2.5)$. Most reported recent anal sex (70.1\%). Approximately $4 \%$ of the sample had ever been diagnosed with anogenital warts.

Less than half of the sample (45.5\%) reported initiating (i.e., receiving at least 1 dose) HPV vaccination Table 1 . Among those who were unvaccinated, $14.2 \%$ were unlikely, $56.3 \%$ were undecided, and $29.4 \%$ likely to in initiate HPV vaccination within the next 12 months.

\section{HPV vaccination}

The bivariate analyses (Table 1) showed that predisposing factors associated with lower prevalence of HPV vaccination were non-Hispanic Black race, lack of college education, and lower vaccine attitudes $(p<0.05)$. Enabling factors associated with lower prevalence of HPV vaccination were lack of health insurance, no past year routine check-up, and no past year STI testing $(p \leq 0.05)$. The need for care factors associated with lower HPV vaccination were never being diagnosed with anogenital warts $(p<0.01)$ and no recent anal sex $(p<0.10)$.

Multivariable analyses are reported in Table 2. Mean vaccine attitudes (predisposing), past year routine check-up (enabling), and ever being diagnosed with anogenital warts (need) were positively associated with a higher probability of HPV vaccination. In the final model, higher vaccine 
Table 1 HPV vaccination and intentions to be vaccinated by predisposing, enabling, and need related factors among Black and Hispanic/Latino sexual and gender minority adults

\begin{tabular}{|c|c|c|c|c|c|c|c|c|}
\hline & \multirow[t]{2}{*}{ Total sample } & \multicolumn{2}{|c|}{$\begin{array}{l}\text { HPV Vaccination } \\
(N=299)\end{array}$} & \multirow[t]{2}{*}{$p$} & \multicolumn{3}{|c|}{$\begin{array}{l}\text { HPV vaccination intentions, next } 12 \text { months } \\
(\mathrm{N}=160)\end{array}$} & \multirow[t]{2}{*}{$p$} \\
\hline & & $\%$ no & $\%$ yes & & $\%$ unlikely & $\%$ undecided & $\%$ likely & \\
\hline Total & & 53.5 & 46.5 & & 14.4 & 56.3 & 29.4 & \\
\hline \multicolumn{9}{|l|}{ Predisposing factors } \\
\hline Mean age (SD) & $26.5(2.7)$ & $26.3(2.72)$ & $26.4(2.68)$ & .48 & $26.7(2.1)$ & $26.7(2.7)$ & $26.5(3.0)$ & .91 \\
\hline Catchup age range & & & & .66 & & & & .53 \\
\hline $18-26$ & $140(46.8)$ & 52.1 & 47.9 & & 11.0 & 58.9 & 30.1 & \\
\hline $27-30$ & $159(53.2)$ & 54.7 & 45.3 & & 17.2 & 54.0 & 28.7 & \\
\hline Ethnoracial identity & & & & .02 & & & & .59 \\
\hline Non-Hispanic Black & $101(33.8)$ & 63.4 & 36.6 & & 10.9 & 57.8 & 31.3 & \\
\hline Hispanic/Latino & $198(66.2)$ & 48.5 & 51.5 & & 16.7 & 55.2 & 28.1 & \\
\hline College educated & & & & .03 & & & & .97 \\
\hline No & $107(35.8)$ & 61.7 & 38.3 & & 15.2 & 56.1 & 28.8 & \\
\hline Yes & $192(64.2)$ & 49.0 & 51.0 & & 13.8 & 56.4 & 29.8 & \\
\hline Sexual identity & & & & .37 & & & & $.72^{\mathrm{a}}$ \\
\hline Gay/same gender loving & $242(80.9)$ & 55.4 & 44.6 & & 14.9 & 56.7 & 28.4 & \\
\hline Bisexual/pansexual & $37(12.4)$ & 43.2 & 56.8 & & 12.5 & 62.5 & 25.0 & \\
\hline Another sexual identity & $20(6.7)$ & 50.0 & 50.0 & & 10.0 & 40.0 & 50.0 & \\
\hline Gender & & & & .55 & & & & $.34^{\mathrm{a}}$ \\
\hline Cisgender man & $279(93.3)$ & 53.1 & 47.0 & & 15.5 & 56.1 & 28.4 & \\
\hline Gender expansive & $20(6.7)$ & 60.0 & 40.0 & & 0.0 & 58.3 & 41.7 & \\
\hline Partnered & & & & .90 & & & & $.22^{\mathrm{a}}$ \\
\hline No & $277(93.0)$ & 53.8 & 46.2 & & 14.8 & 54.4 & 30.9 & \\
\hline Yes & $21(7.1)$ & 52.4 & 47.6 & & 9.1 & 81.8 & 9.1 & \\
\hline COVID-19 vaccination intentions & & & & .74 & & & & $.05^{\mathrm{a}}$ \\
\hline Unlikely/Undecided & $43(14.4)$ & 51.2 & 48.8 & & 31.8 & 40.9 & 27.3 & \\
\hline Likely & 256 (85.6) & 53.9 & 46.1 & & 11.6 & 58.7 & 29.7 & \\
\hline Mean vaccine attitudes scale (SD) & $3.6(0.5)$ & $3.5(0.5)$ & $3.7(0.5)$ & $<.01$ & $3.6(0.5)$ & $3.5(0.4)$ & $3.4(0.6)$ & .18 \\
\hline \multicolumn{9}{|l|}{ Enabling Factors } \\
\hline Health insurance & & & & .04 & & & & .32 \\
\hline No & $46(15.4)$ & 67.4 & 32.6 & & 12.9 & 67.7 & 19.4 & \\
\hline Yes & $253(84.6)$ & 51.0 & 49.0 & & 14.7 & 53.5 & 31.8 & \\
\hline Past year routine checkup & & & & $<.01$ & & & & .11 \\
\hline No & $118(39.5)$ & 65.3 & 34.8 & & 18.2 & 59.7 & 22.1 & \\
\hline Yes & $181(60.5)$ & 45.9 & 54.1 & & 10.8 & 53.0 & 36.1 & \\
\hline STI test within past year & & & & .05 & & & & .58 \\
\hline No & $67(22.4)$ & 64.2 & 35.8 & & 16.3 & 60.5 & 23.3 & \\
\hline Yes & $232(77.6)$ & 50.4 & 49.6 & & 13.7 & 54.7 & 31.6 & \\
\hline PrEP Use & & & & .11 & & & & .29 \\
\hline Never & $109(37.2)$ & 59.6 & 40.4 & & 15.4 & 55.4 & 29.2 & \\
\hline Yes, not current & $60(20.5)$ & 55.0 & 45.0 & & 24.2 & 57.6 & 18.2 & \\
\hline Yes, current & $124(42.3)$ & 46.0 & 54.0 & & 8.8 & 59.7 & 31.6 & \\
\hline \multicolumn{9}{|l|}{ Need based factors } \\
\hline Diagnosed with anogenital warts & & & & $<.01$ & & & & \\
\hline No & $286(95.7)$ & 55.2 & 44.8 & & b & & & \\
\hline Yes & $13(4.4)$ & 15.4 & 84.6 & & & & & \\
\hline Anal sex, past month & & & & .07 & & & & .90 \\
\hline No & $89(29.9)$ & 61.8 & 38.2 & & 12.7 & 58.2 & 29.1 & \\
\hline Yes & $209(70.1)$ & 50.2 & 49.8 & & 15.2 & 55.2 & 29.5 & \\
\hline Mean age at first anal sex with a man (SD) & $17.6(2.5)$ & $17.7(2.5)$ & $17.4(2.5)$ & .22 & $17.7(2.5)$ & $17.9(2.6)$ & $17.6(2.6)$ & .79 \\
\hline
\end{tabular}

Bolded entries are statistically significant at $p<0.05$

${ }^{\mathrm{a}}$ Fisher exact test

${ }^{\mathrm{b}}$ Cell sizes too small to estimate 
Table 2 Predisposing, enabling, and need related factors associated with HPV vaccination among Black and Hispanic/Latino sexual and gender minority adults

\begin{tabular}{|c|c|c|c|c|}
\hline & Predisposing factors & Enabling factors & Need for care & $\begin{array}{l}\text { Independent predis- } \\
\text { posing and enabling } \\
\text { factors }\end{array}$ \\
\hline & $\begin{array}{l}\text { Model } 1 \\
\text { aPR }(95 \% C I)\end{array}$ & $\begin{array}{l}\text { Model } 2 \\
\text { aPR }(95 \% C I)\end{array}$ & $\begin{array}{l}\text { Model } 3 \\
P R(95 \% C I)\end{array}$ & $\begin{array}{l}\text { Model } 4 \\
\text { aPR }(95 \% C I)\end{array}$ \\
\hline \multicolumn{5}{|l|}{ Race } \\
\hline Non-Hispanic Black & 1.00 & & & \\
\hline Hispanic/Latino & $1.23(0.92-1.58)$ & & & \\
\hline \multicolumn{5}{|l|}{ College educated } \\
\hline No & 1.00 & & & \\
\hline Yes & $1.21(0.92-1.59)$ & & & \\
\hline Mean vaccine attitudes scale & $1.51(1.07-1.59)$ & & & $1.55(1.12-2.15)$ \\
\hline \multicolumn{5}{|l|}{ Health insurance } \\
\hline No & & 1.00 & & \\
\hline Yes & & $1.51(0.98-2.31)$ & & \\
\hline \multicolumn{5}{|l|}{ Past year routine checkup } \\
\hline No & & 1.00 & & 1.00 \\
\hline Yes & & $1.47(1.10-1.95)$ & & $1.50(1.14-1.97)$ \\
\hline \multicolumn{5}{|l|}{ STI test within past year } \\
\hline No & & 1.00 & & \\
\hline Yes & & $1.26(0.89-1.78)$ & & \\
\hline \multicolumn{5}{|l|}{ Diagnosed with anogenital warts } \\
\hline No & & & 1.00 & 1.00 \\
\hline Yes & & & $1.78(1.36-2.35)$ & $1.48(1.15-1.90)$ \\
\hline \multicolumn{5}{|l|}{ Anal sex, past month } \\
\hline No & & & 1.00 & \\
\hline Yes & & & $1.24(0.92-1.68)$ & \\
\hline
\end{tabular}

$a P R$, adjusted prevalence ratios; $P R$, crude prevalence ratio

aPRs in bold indicate statistical significance $>0.05$. Regression results for the HPV vaccine intentions outcome are reported in the "Results" section

attitudes $(a P R=1.55 ; 95 \% C I: 1.12-2.15)$, past year routine check-up $(a P R=1.50 ; 95 \% C I: 1.14-1.97)$, and ever being diagnosed with anogenital warts $(a P R=1.55 ; 95 \% C I$ : 1.12-2.15) were independently associated with the probability of HPV vaccination.

\section{HPV vaccination intentions}

In bivariate analyses, only COVID-19 vaccination intention was associated with HPV vaccination intentions $(p=0.05)$; however, COVID-19 vaccine intentions (likely vs undecided) unlikely) were not associated with HPV vaccine intentions in regression models (HPV undecided vs unlikely $[P R=1.48$; 95\% CI: 0.96-2.31] or (HPV likely vs. unlikely $[P R=1.56$; 95\% CI: 02.87]).

\section{Discussion}

In this study HPV vaccination was underutilized by Black and Hispanic MSM. Nearly half of the sample was within the recommended age range for routine catchup vaccination and the other half had aged out within the previous 4 years [7]. Yet, more than half remained unvaccinated despite the presence of multiple enabling factors such as having health insurance, having a past year medical check-up, undergoing recent STI testing, and having been on PrEP. Among these enabling factors, only a past year routine check-up was associated with a higher probability of HPV vaccination.

The vaccine attitudes, but not race or educational attainment, was the predisposing factor independently associated with HPV vaccination. This is in contrast to previous research finding college education significantly associated with HPV vaccination $[10,16]$. However, in our 
model, the effect of education was mitigated by vaccine attitudes. Higher levels of educational attainment were also positively associated with vaccine attitudes in the general US population [17]. Improving attitudes toward vaccinations-particularly among MSM with less education-should be a part of the efforts to increase reach and access to HPV vaccination specifically and information about anal cancer prevention more generally $[18,19]$.

Analysis of need related factors showed that a history of HPV-related disease (i.e., lifetime occurrence of anogenital warts) was associated with HPV vaccination; however, recent risk behaviors (i.e., anal sex) were not associated with HPV vaccination. This suggests that, at least in some cases, the recommendation and uptake for HPV vaccination is reactionary to the presence of clinical disease. HPV vaccine status should be assessed at healthcare visits involving STI testing and PrEP initiation to increase reach and equity for ethnoracial MSM. The findings reported here reflect patterns of missed opportunities for HPV vaccination among MSM engaged in sexual healthcare [10].

Findings from this study are limited by a few key methodological considerations. First, HPV vaccination was self-reported. While recall of HPV vaccination was found to be highly sensitive among adult MSM, bias is likely higher among those who were vaccinated at younger ages [20]. Second, the non-probability sampling utilized in acquiring this sample may limit the generalizability of the findings. The sample was mostly college educated and had high levels of healthcare access and utilization. Additional barriers likely exist for those with less socioeconomic resources. Lastly, this sample consisted mostly of cisgender MSM. More research is needed to better understand barriers to HPV vaccination among transgender women and gender-expansive natal males.

Targeted efforts are needed to reduce anal cancer disparities experienced by ethnoracial minority MSM. Promoting HPV vaccination through culturally targeted mobile phone interventions may help to modify predisposing factors (e.g., vaccine attitudes) and can be modeled after existing sexual health interventions [21,22]. Similarly, social media campaigns targeting Black and Hispanic MSM have been effective at promoting awareness and positive attitudes toward PrEP and can be adapted to promote HPV vaccination and anal cancer prevention more broadly [23].

Interventions should also leverage existing enabling factors, such as the utilization of sexual health services (e.g., PrEP) in which HPV vaccination is not currently being routinely promoted [24]. For example, HPV vaccine status should be routinely assessed for MSM during sexual health visits involving STI testing and PrEP management. Targeting providers with anal cancer prevention education, clinic-based feedback, and communication strategies has been shown to improve HPV vaccination in the general population and can be adapted specifically for clinics with large MSM patient populations [24, 25]. HPV vaccination among ethnoracial minority MSM is an under-explored area of research and requires further intervention-focused research.

Acknowledgements The authors acknowledge the Human Rights Campaign, in particular Charleigh Flohr and Ellen Kahn, for their contributions to the collection of data for this project.

Author Contribution All authors contributed to the study conception and design. Material preparation and data collection were performed by Ryan Watson and Lisa Eaton. Data analysis was performed by Christopher Wheldon and Ryan Watson. The first draft of the manuscript was written by Christopher Wheldon and all authors commented on previous versions of the manuscript. All authors read and approved the final manuscript.

Funding The National Institutes of Drug Abuse grant K01DA047918.

Data Availability The data is not publicly available.

Code Availability SAS code is available from Dr. Wheldon.

\section{Declarations}

Ethics Approval All study protocols were approved by the University of Connecticut's Institutional Review Board.

Consent to Participate All participants received informed consent. A waiver of documented consent was approved by the University of Connecticut's Institutional Review Board.

Consent for Publication Participants provided consent about the reporting of research results.

Disclaimer The opinions expressed in this article are the author's own and do not reflect the view of the National Institutes of Health.

Conflict of Interest The authors declare no competing interests.

\section{References}

1. Grulich AE, Poynten IM, Machalek DA, Jin F, Templeton DJ, Hillman RJ. The epidemiology of anal cancer. Sex Health. 2012;9:504.

2. Cruz A, Chen D, Hsu P, Pandit V, Omesiete P, Vij P, et al. Racial and gender disparities in the incidence of anal cancer: analysis of the Nationwide Inpatient Sample (NIS). J Gastrointest Oncol. 2019;10:37-41.

3. Bojko MM, Kucejko RJ, Poggio JL. Racial disparities and the effect of county level income on the incidence and survival of young men with anal cancer. Health Equity. 2018;2:193-8.

4. Walsh T, Bertozzi-Villa C, Schneider JA. Systematic review of racial disparities in human papillomavirus-associated anal dysplasia and anal cancer among men who have sex with men. Am J Public Health. 2015;105:e34-45. 
5. Lin D, Gold HT, Schreiber D, Leichman LP, Sherman SE, Becker DJ. Impact of socioeconomic status on survival for patients with anal cancer. Cancer. 2018;124:1791-7.

6. Bucher G, Torrence A, Schneider J, Houlberg M, Munar D, Keglovitz KB. Anal dysplasia screening guidelines. 2019. https:// howardbrown.org/wp-content/uploads/2019/08/Howard_Brown_ Health_Anal_Cancer_Screening_Guidelines-2019.pdf. Accessed 1 Sept 2021

7. Meites E, Szilagyi PG, Chesson HW, Unger ER, Romero JR, Markowitz LE. Human Papillomavirus vaccination for adults: updated recommendations of the Advisory Committee on Immunization Practices. 2019;68:698-702.

8. Food and Drug Administration. Prescribing information [package insert]. Gardasil 9 (human papillomavirus 9-valent vaccine, recombinant). [Internet]. Silver Spring, MD: US Department of Health and Human Services, Food and Drug Adiminstration; 2018. Available from: https://www.fda.gov/media/90064/downl oadexternal. Accessed 1 Sept 2021

9. Elam-Evans LD, Yankey D, Singleton JA, Sterrett N, Markowitz LE, Williams CL, et al. National, regional, state, and selected local area vaccination coverage among adolescents aged 13-17 years - United States, 2019. MMWR Morb Mortal Wkly Rep. 2020;69:1109-16.

10. McClung N, Burnett J, Wejnert C, Markowitz LE, Meites E. Human papillomavirus vaccination coverage among men who have sex with men-National HIV Behavioral Surveillance, United States, 2017. Vaccine. 2020;38:7417-21.

11. Pho AT, Mangal S, Bakken S. Human papillomavirus vaccination among transgender and gender diverse people in the United States: an integrative review. Transgender Health. 2021; ahead of print. https://doi.org/10.1089/trgh.2020.0174.

12. Damaskos P, Amaya B, Gordon RA, Walters CB. Intersectionality and the LGBT cancer patient. Seminars in Oncology Nursing. Elsevier Inc.; 2018;34:30-6.

13. Nadarzynski T, Frost M, Miller D, Wheldon CW, Wiernik BM, Zou H, et al. Vaccine acceptability, uptake and completion amongst men who have sex with men: a systematic review, metaanalysis and theoretical framework. Vaccine. 2021;39:3565-81.

14. Andersen RM. Revisiting the Behavioral Model and Access to Medical Care: Does it Matter? J Health Soc Behav. 1995;36:1-10.

15. Hamilton CM, Strader LC, Pratt JG, Maiese D, Hendershot T, Kwok RK, et al. The PhenX Toolkit: get the most from your measures. Am J Epidemiol. 2011;174:253-60.
16. Meites E, Krishna NK, Markowitz LE, Oster AM. Health care use and opportunities for human papillomavirus vaccination among young men who have sex with men. Sex Transm Dis. 2013;40:154-7.

17. Lin F-Y, Wang C-H. Personality and individual attitudes toward vaccination: a nationally representative survey in the United States. BMC Public Health. 2020;20:1759.

18. Wheldon CW, Maness SB, Islam JY, Deshmukh AA, Nyitray AG. Gay and bisexual men in the US lack basic information about anal cancer. J Low Genit Tract Dis. 2021;25:48-52.

19. Wheldon CW, Daley EM, Buhi ER, Baldwin JA, Nyitray AG, Giuliano AR. HPV vaccine decision-making among young men who have sex with men. Health Educ J. 2017;76:52-65.

20. Forward T, Meites E, Lin J, Hughes JP, Unger ER, Markowitz LE, et al. Sensitivity of self-reported HPV vaccination history among 18-26 year-old men who have sex with men - Seattle, Washington, 2016-2018. Sexually Transmitted Diseases. 2021;Publish Ahead of Print.

21. Reiter PL, Katz ML, Bauermeister JA, Shoben AB, Paskett ED, McRee A-L. Increasing human papillomavirus vaccination among young gay and bisexual men: a randomized pilot trial of the Outsmart HPV Intervention. LGBT Health. 2018;5:325-9.

22. Fish J, Papaloukas P, Jaspal R, Williamson I. Equality in sexual health promotion: a systematic review of effective interventions for black and minority ethnic men who have sex with men. BMC Public Health. 2016;16:810.

23. Kudrati SZ, Hayashi K, Taggart T. Social Media \& PrEP: A systematic review of social media campaigns to increase PrEP awareness \& uptake among young Black and Latinx MSM and women. AIDS Behav. 2021;25:4225-34.

24. Wheldon CW, Sutton SK, Fontenot HB, Quinn GP, Giuliano AR, Vadaparampil ST. Physician communication practices as a barrier to risk-based HPV vaccine uptake among men who have sex with men. J Canc Educ. 2018;33:1126-31.

25. Krantz L, Ollberding NJ, Beck AF, Carol BM. Increasing HPV vaccination coverage through provider-based interventions. Clin Pediatr. 2018;57:319-26.

Publisher's Note Springer Nature remains neutral with regard to jurisdictional claims in published maps and institutional affiliations. 